\title{
Gastric cancer: Metabolic and metabolomics perspectives (Review)
}

\author{
SHIYU XIAO and LIYA ZHOU \\ Department of Gastroenterology, Peking University Third Hospital, \\ Haidian, Beijing 100191, P.R. China
}

Received March 17, 2017; Accepted May 2, 2017

DOI: 10.3892/ijo.2017.4000

\begin{abstract}
Gastric cancer is one of the most malignant tumors worldwide and remains a major health threat in Asia-Pacific regions, while its pathological mechanism is generally unknown. Recent research has advanced the understanding of the relationship between metabolic reprogramming and carcinogenesis. In particular, metabolic regulation and cancer research are being further brought into sharp focus with the emergence of metabolomics. Not only can metabolomics provide global information on metabolic profiles of specific tumors, but it can also act as a promising tool to discover biomarkers regarding diagnosis, metastatic surveillance and chemotherapeutic sensitivity prediction. Meanwhile, metabolism-based anticancer therapies will be further discovered. Up to now, accumulative studies have highlighted the application of metabolomics in gastric cancer research regarding different aspects; therefore we summarized the current available results of how metabolic changes are linked to gastric carcinogenesis, and how metabolomics holds promise for the diagnosis, metastatic surveillance, treatment and prognosis prediction of gastric cancer.
\end{abstract}

\section{Contents}

1. Introduction

2. Metabolic alteration in gastric cancer

3. Metabolomics in diagnosis, treatment and prognostic prediction of gastric cancer

4. Current perspectives and future directions

5. Conclusions

Correspondence to: Professor Liya Zhou, Department of Gastroenterology, Peking University Third Hospital, 49 North Huayuan Road, Haidian, Beijing 100191, P.R. China

E-mail: zhouly_bjmu@163.com

Key words: gastric cancer, metabolomics, metabolic reprogramming, diagnosis, metastatic prediction, treatment

\section{Introduction}

Gastric cancer remains third in ranking in cancer death worldwide, although its overall incidence is declining in recent years (1). In the past decades, studies aimed at Helicobacter pylori infection $(2,3)$, hereditary susceptibility (4) and environmental factors (5) have made a great breakthrough in investigating its precise pathogenesis. Recently, application of various '-omics' technologies opened a new field to investigate the mechanisms behind this disease.

With the emergency of metabolomics, major progress has been made in the understanding of the relationship between metabolic regulation and cancer. Warburg, in fact, showed a characteristic metabolic pattern of tumors in the 1920s, that is, tumor cells consume a large amount of glucose for glycolysis even under the condition of sufficient oxygen (Warburg effect) (6). Extensive research also indicates that metabolic reprogramming is one of the hallmarks of cancer (7), and intricately linked to oncogenesis (8-10) and cancer immune escape (11-13). On the other hand, study methods combined conventional oncology research and metabolomics are more likely to provide deeper insights in this field. The procedure of these methods is illustrated in Fig. 1, and more detailed information can be found in literature (14-16).

Several excellent reviews have been published on metabolomics application in different diseases (17-19) especially cancer research (20-23). Hence, this report presents fresh and profound insights into metabolic changes in gastric cancer and possible mechanism behind these alterations is further discussed. Then, we focus on some studies including our data targeted on biomarkers involving diagnosis, metastasis and prognosis, and treatment in this disease. Finally, future directions are presented.

\section{Metabolic alteration in gastric cancer}

Up to now, several studies aimed at identifiable metabolic changes in macroenvironment-blood (24-29) (Table I) and urine (30-34) (Table II) or microenvironment-carcinoma tissues (35-41) (Table III) and gastric juice (42-44) (Table IV) have been done to map globally metabolic profiles and interpret its possible mechanism in the process of gastric carcinogenesis. Typical changes in metabolites of this disease are illustrated in Fig. 2. 


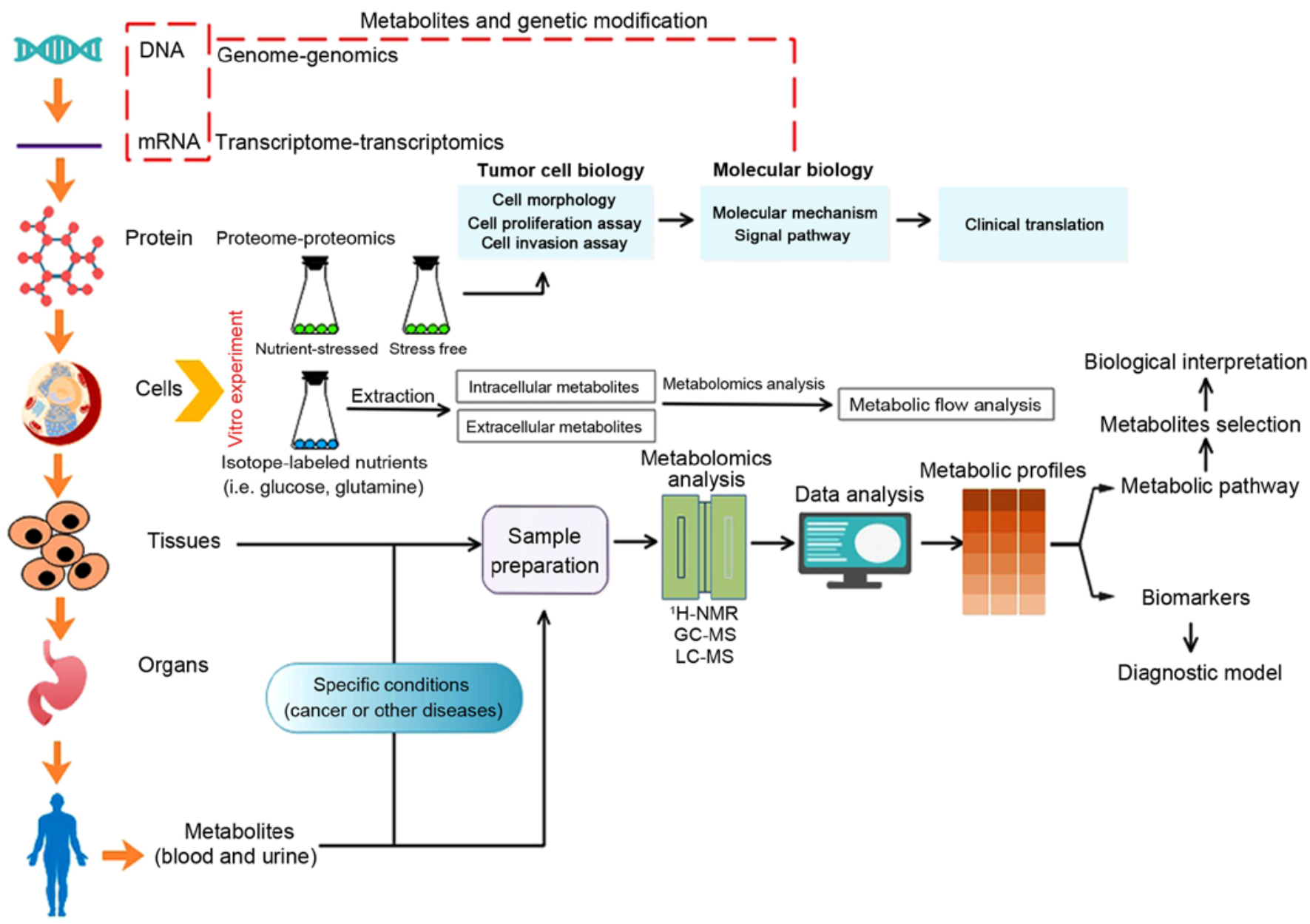

Figure 1. Metabolism and metabolomics in cancer research. Concerning tumor cell lines cultured in vitro, either conventional cell biology research or isotopic tracer experiment is available. Tumor cytobiological methods (cell morphology, cell proliferation assay and cell invasion assay) can be utilized to assess cytobiological behaviors under specific nutrient-stressed or stress-free condition, and further investigations targeted at precise mechanism and significance can be confirmed via molecular biology techniques. With regard to isotopic tracer experiment, the flow of nutrients and metabolites can be identified with isotopic tracer, then the significance of specific nutrients or metabolites and its potential divergent fates toward meeting the demands of either energetic utilization or synthesizing macromolecules in cancer cells can be identified (14). Metabolites that are different between tumor groups and control groups are able to be detected through metabolomics analysis (such as ${ }^{1} \mathrm{H}-\mathrm{NMR}$, ${ }^{1}$ hydrogen-nuclear magnetic resonance; LC-MS, liquid chromatography-mass spectrometry; GC-MS, gas chromatography-mass spectrometry) and data analysis, metabolic biomarkers or metabolic pathway that is specific to certain cancers were discovered to benefit cancer research $(15,16)$. Of note, combination of genomics, transcriptomics and proteomics plus metabolomics can further give us comprehensive understanding of cancers toward systematic biology.

Glucose metabolism. Cumulative evidence demonstrates that concentration of lactic acid shows a consistent increase in urine (30) or tissue $(31,35,36,38)$ samples of gastric cancer groups, but glucose is considerably depleted compared with those healthy counterparts or non-malignant patients (like chronic superficial gastritis and chronic atrophic gastritis without intestinal metaplasia) $(35,36)$. The high lactate level might be attributed to the special metabolism of most cancer cells, known as 'Warburg effect' we mentioned above (6). Scarce glucose might result from the overexpression of glucose transporters (42) and type II hexokinase (43), which are both confirmed in gastric cancer tissues. Higher fructose-6-phosphokinase (6-FPK) activity can also result in low glucose in gastric cancer tissues (44), as it regulates the output of glucose to glycolysis pathway. The glycolytic switch has been identified to be associated with oncogenic transformation and molecular signal transduction, such as hypoxia-inducible factor pathway, insulin signaling pathway and PI3K-Akt-mTOR pathway (45). Furthermore, over- expression of pyruvate kinase and lactate dehydrogenase is positively associated with tumor proliferation and poor prognosis, downregulation of them in vitro experiment can impair tumor invasion $(38,46-49)$. On the other hand, such special microenvironment might be the requirement of rapid propagation of tumor cells. To our understanding, it has been reported that accumulated lactic acid moderates the activity of proteases that decompose extracellular matrix, which can produce some peptides and amino acids that are consumable for energy generation (44). Acidosis microenvironment is also ascribed to the formation of cancer blood vessels, meeting the plentiful supply of nutrients and leading to tumor invasion and metastasis (50). Moreover, tumor-derived lactate shows strongly negative effects on cytotoxic T-cell/NK cell function $(11,51)$ and blocks differentiation of monocytes to dendritic cells (52), finally leading to tumor immune escape. However, such outcome demands further verification in gastric cancer.

Considering tricarboxylic acid cycle (TCA) intermediates, an increase of five metabolites ( $\alpha$-ketoglutaric acid, malic acid, 


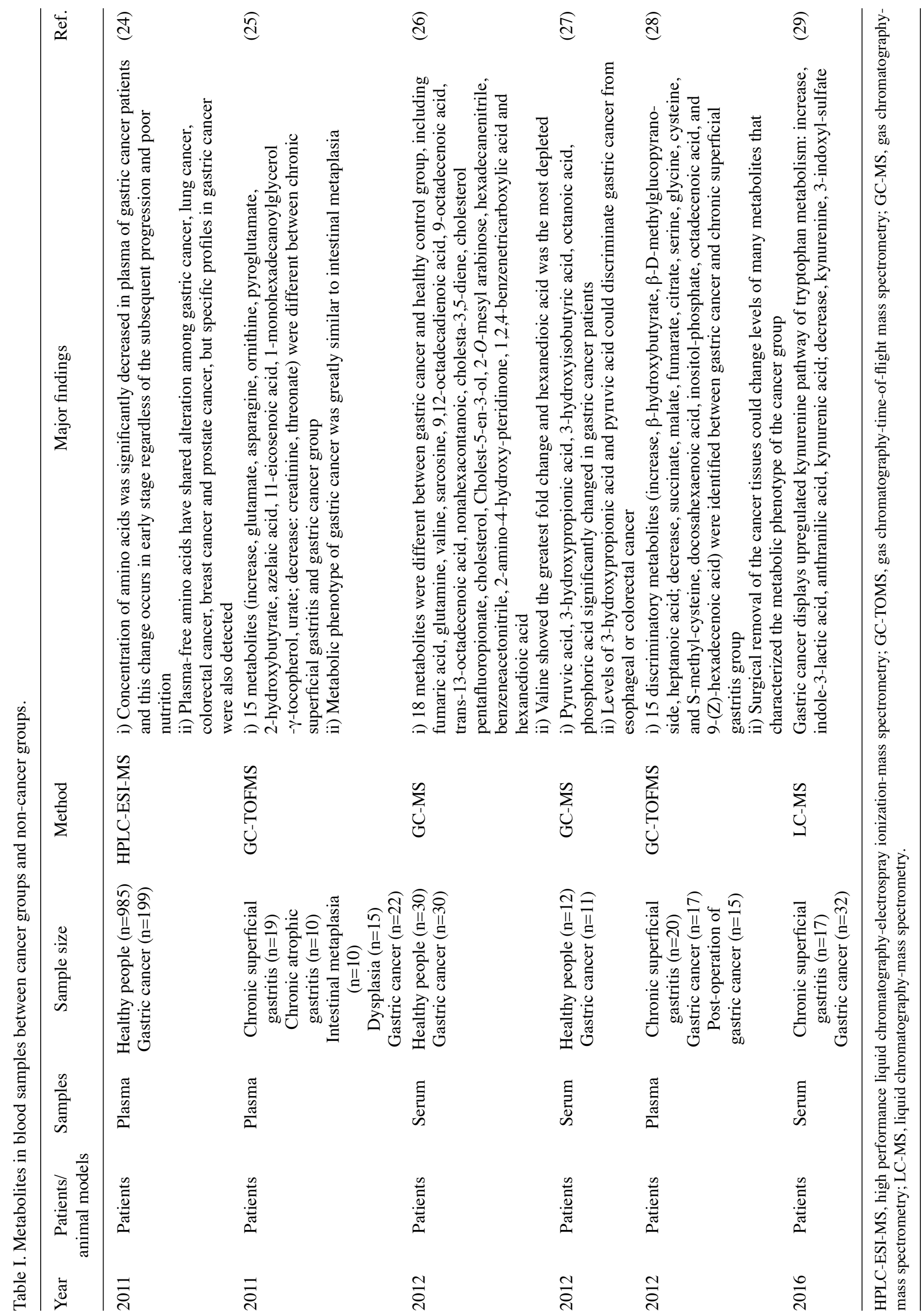




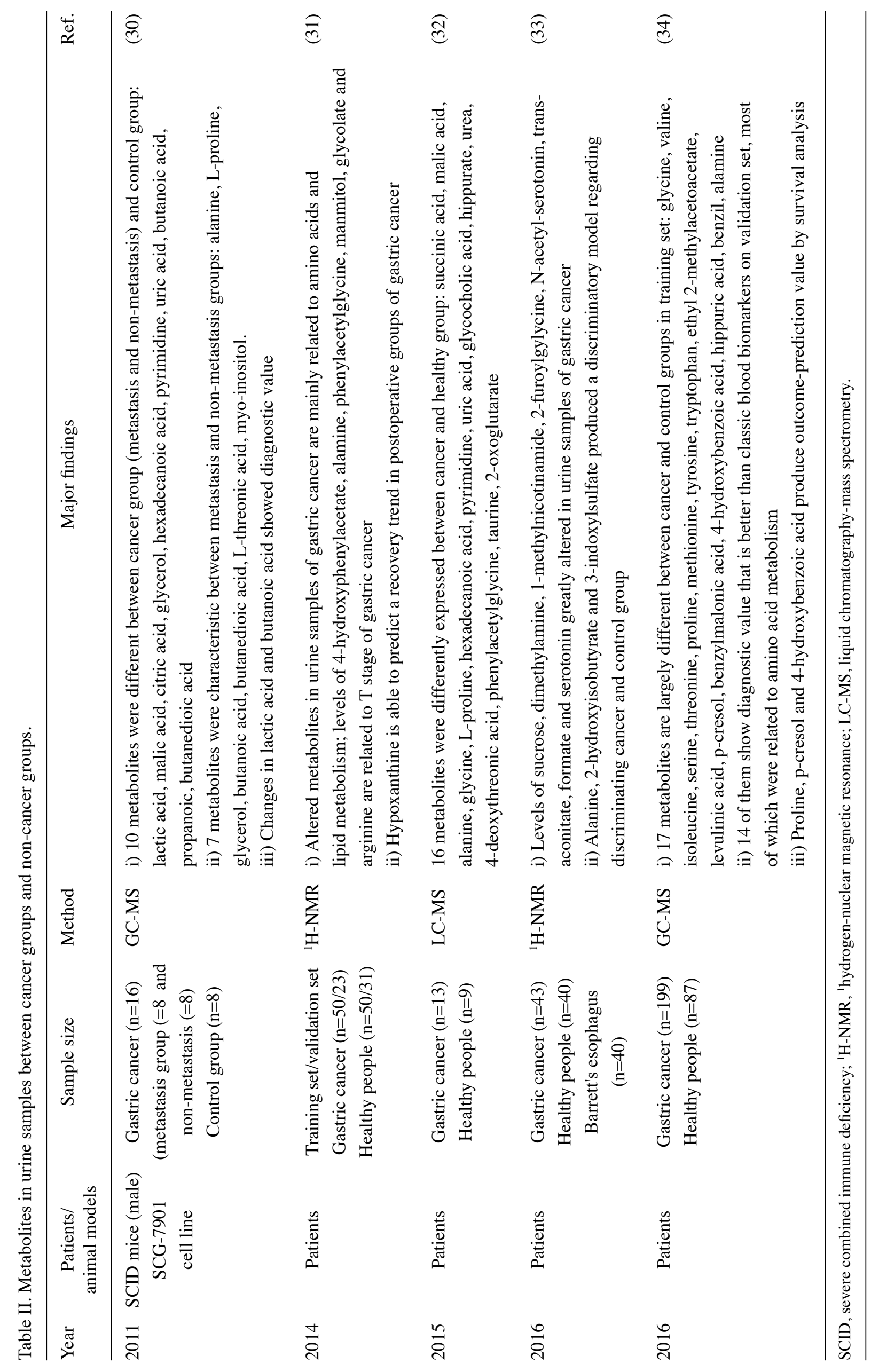




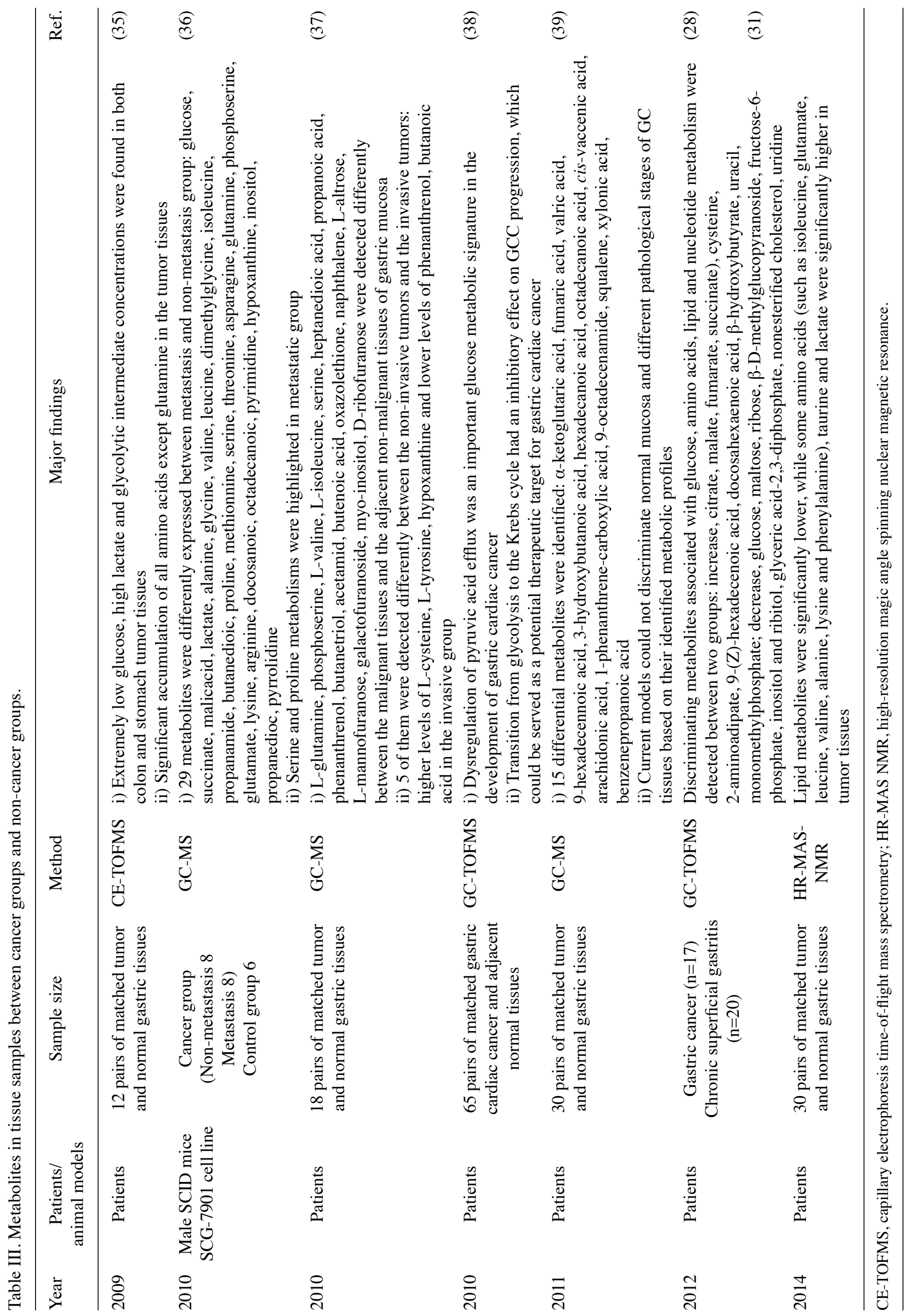


fumarate, succinate, citric acid) is noticed regardless of blood $(26,28)$, urine $(30,32)$ or tissue $(28,35,36,39)$ samples in gastric cancer. There are some possible reasons that can explain this phenomenon. One account is that cancer cells still use a small portion of glucose for oxidative phosphorylation. Secondly, cancer cells might also utilize fumarate respiration to generate energy under special conditions of glucose deprivation and severe hypoxia in microenvironment (53), and succinate is one of the byproducts in this process except for originating from TCA. Hence, it provides a likely explanation for the accumulation of fumarate and succinate. Another reason is that some amino acids, such as glutamine, threonine, phenylalanine, tyrosine or proline, can be converted into these intermediates involving in TCA (Fig. 2). Additionally, elevated levels of citric acid can be used in the de novo fatty acid synthesis, but it is noted that citrate can also induce apoptosis in two gastric cancer cell lines in vitro experiment $(54,55)$.

Amino acid metabolism. Availability of amino acids is pivotal for cellular protein biosynthesis and cytoskeleton formation, while it has been pointed out that amino acids especially those linking to TCA (Fig. 2) are an alternative energy source of cancer cell proliferation (56). By employing metabolomics technologies, levels of various amino acids (including serine, valine, phenylalanine, tryptophan, glycine, and proline) and their primary derivatives (such as kynurenine, kynurenic acid, anthranilic acid and nicotinic acid) are significantly higher in tissue specimens $(31,36,37)$ and gastric content $(29,40,41)$, but decreased concentration in some of them is observed in blood (24). The overexpression of L-type amino acid transporter 1 (LAT1) might be proposed to explain this dissimilarity (57). Free amino acids are greatly assimilated to cancer tissues via LAT1 from bloodstream, resulting in the low accumulation of amino acid in contrast to normal counterparts. Malnourishment may also be a contributing factor to these reduced levels of plasma amino acids. Apart from these, degradation of extracellular matrix mediated by the overexpressed matrix metalloproteinases (MMPs) and activated autophagic degradation of intracellular proteins are considered as the potential source of accumulative amino acids in tumor tissues (58-60).

Elevated amino acids in microenvironment are contributing factors in carcinogenesis. Most strikingly, it is indicated that many cancer cell lines cannot survive in the absence of glutamine (61), because it is required for anabolic growth of mammalian cells through its ability to control the master regulator of protein translation mTORC1 (62). Reprogramming of glutamine metabolism further contributes to the proliferative and metabolic responses regulated by oncogenic transcription factor c-MYC (63). In addition, it is also the nitrogen donor for several key metabolic enzymes and for the de novo synthesis of both purines and pyrimidines (Fig. 2). Serine also participates in the de novo synthesis of nucleotides by serving one carbon unit. Functional genomics further indicates that serine biosynthesis pathway is significant for breast cancer event, which can be attributable to the overexpression of phosphoglycerate dehydrogenase (PHGDH) that controls the flow of intermediates originated from glycolysis (64). Inhibition of PHGDH in cells can result in lower serine and decrease cellular proliferation in vitro. However, this remains 


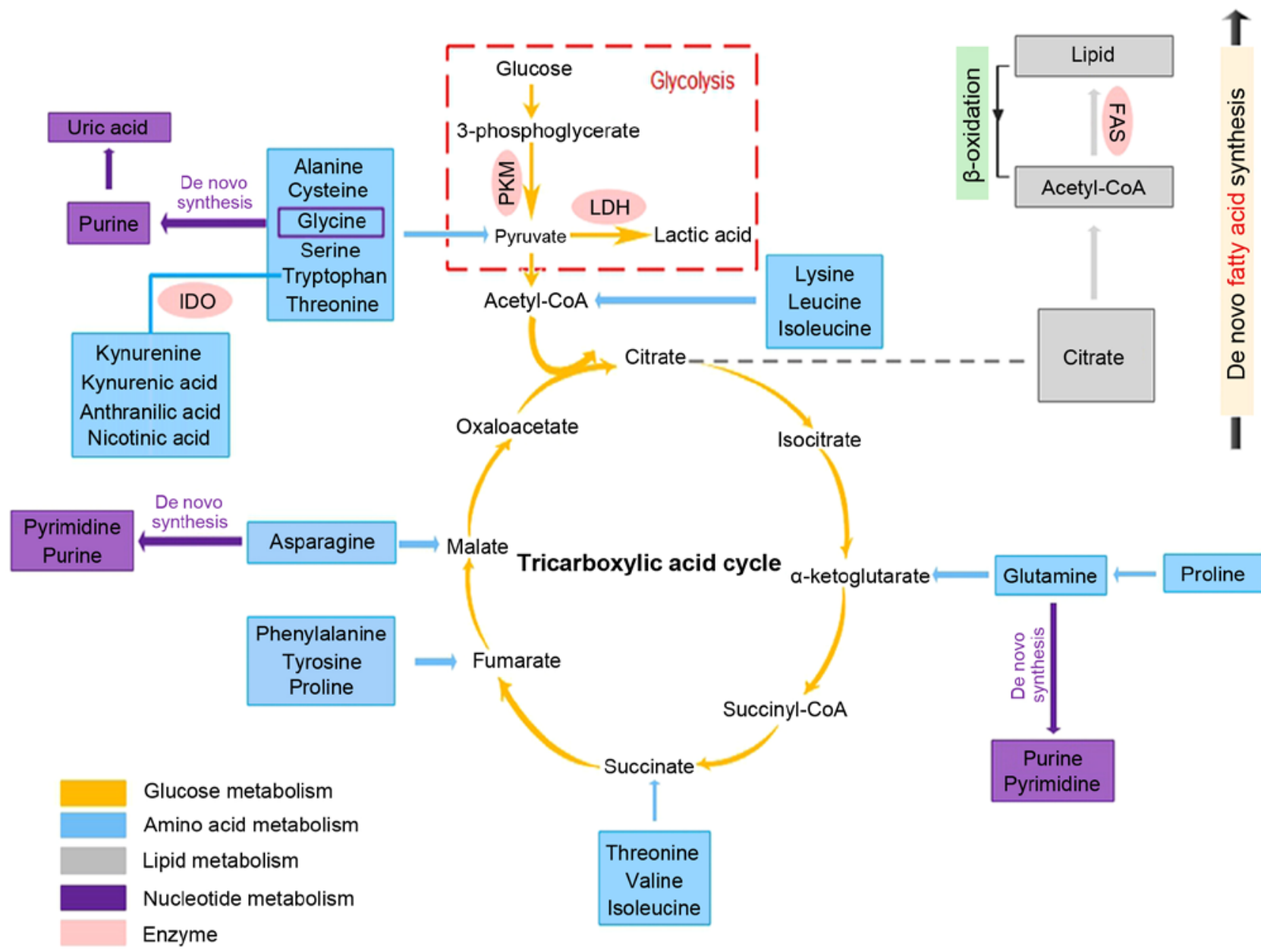

Figure 2. Metabolic regulation in gastric cancer. Altered metabolites in gastric can be categorized into four main biomolecules: carbohydrates, amino acids, lipids and nucleic acids. Activated glycolysis and impaired aerobic respiration shape the altered glucose metabolism in this disease. For amino acid metabolism, various amino acids (serine, valine, phenylalanine, tryptophan, glycine, and proline) and some primary derivatives (such as kynurenine, kynurenic acid, anthranilic acid and nicotinic acid) are significantly higher in tissue specimens and gastric content, but decreased concentration is observed in blood samples. Of note, glutamine is also the most greatly depleted. Increased rate of lipogenesis, upregulation of fatty acid $\beta$-oxidation and upregulated oxidative degradation are the typical characteristics of lipid metabolism in this disease. Accumulation of the end products of nucleotide catabolism is characterized by the higher levels of uric acid. Moreover, there is correlation between these four metabolisms. For instance, glycine, asparagine and glutamine are used as building blocks of purines.

unclear in gastric cancer. Tryptophan and its downstream metabolites (mainly including kynurenine, kynurenic acid, anthranilic acid, nicotinic acid) via kynurenine pathway are related to the pathogenesis and prognosis of various malignancies including gastric cancer $(65,66)$. Kynurenine pathway catalyzed by indoleamine-2, 3-dioxygenase (IDO) plays a key role in adapting the tumor microenvironment to favor cancer progression because higher IDO expression is associated with an increase in immunosuppressive T-regulatory cell activity (67), and its immunosuppressive role inhibits T-cell mediated cytotoxicity and cell proliferation of gastric cell lines in vitro (68). Additionally, 3-hydroxyanthanilie acid (downstream metabolites in kynurenine pathway) also has suppressive effects on inflammation and immune response (69). Glycine used in living organism as building blocks of purines is strongly correlated with the rapid proliferation rates, and then antagonizing glycine uptake and its mitochondrial biosynthesis preferentially impair rapidly proliferating cells (70). The indirect anti-angiogenic impact of glycine is also identified in vitro $(71,72)$ and in vivo $(73,74)$, possibly because it might inhibit the proliferation of vascular endothelial cells, finally leading to angiogenesis (74). Elevated proline in tumor tissues might begin with the activation of MMPs and degradation of microenvironmentally extracellular matrix (ECM), subsequently the degradation of collagen catalyzed by proline dehydrogenase (PRODH) that can be regulated under conditions of nutrient stress linked to mTOR signaling system (75). Other elevated amino acids, such as tyrosine, valine and cysteine, can be converted into the TCA intermediates (except for citric acid, isocitrate, succinyl-CoA, oxaloacetate) to generate energy (Fig. 2).

Lipid metabolism. The notable feature of lipid metabolism in cancer cells is an increased rate of lipogenesis and the upregulation of mitochondrial fatty acid $\beta$-oxidation, gastric cancer shows a similar tendency and presents typical changes regarding various metabolites involving in lipid metabolism.

Fatty acids, such as hexadecenoic acid, docosahexaenoic acid, eptanoic acid and $\beta$-hydroxybutyrate, are significantly larger in gastric cancer tissues than in benign tissues (like 
chronic superficial gastritis) (28). Octadecanoic acid is also found to be elevated in blood specimens obtained from gastric cancer patients (39). Of them, $\beta$-hydroxybutyrate is the common product of fatty acid degradation via $\beta$-oxidation, suggesting more intensive decomposition of fatty acids in microenvironment. The accelerated metabolism from lipids to fatty acids and finally ketone bodies consumes fat, which might explain the fact that patients become very thin in later stages of gastric cancer. This signature also has been identified by the xenograft animal models with gastric cancer showing elevated levels of glycerol and hexadecanoic acid (30), resulting from the high activation of adipocyte lipolysis in cancer cells as well as enhanced expression and function of adipocyte hormone-sensitive lipase in cancer cachexia (76). In contrast, some data show that unsaturated fatty acids such as 9-hexadecenoic acid, cis-vaccenic acid, arachidinic acid, hexadecanoic acid and 3-hydroxybutanoic acid are found to be significantly decreased in cancer tissue samples (26). Of note, the level of $O$-acetylcarnitine, which increases the $\beta$-oxidation of fatty acid, shows a declining trend as the early gastric cancer progresses into advanced stage (31). Accordingly, it seems that decreased $O$-acetylcarnitine might explain the impaired fatty acids $\beta$-oxidation in stage III/IV gastric cancer, which is characterized by the decline in unsaturated fatty acids we discussed above (9-hexadecenoic acid, cis-vaccenic acid, arachidinic acid, hexadecanoic acid and 3-hydroxybutanoic acid). However, this discrepancy between different research needs further elucidation with larger samples and different analytical methods. On the other hand, free fatty acids in plasma, including palmitic acid, stearic acid, 9-(Z)-hexadecenoic acid, oleic acid, linoleic acid, docosahexaenoic acid and arachidonic acid, are equivalent in both gastric cancer and gastric benign disorders (25). Therefore, it infers that free fatty acids in blood might be not utilized by tumor cells.

Upregulated lipid peroxides are also confirmed in this disease. Accumulation of 4-hydroxyphenylacetate resulting from the oxidative degradation of lipids was observed in the study of Jung et al (31). Elevation of azelaic acid in blood samples, which is the end product of linoleic acid when subjected to peroxide decomposition (77) and can serve as a marker of lipid peroxidation (78), as observed by Yu et al (25).

Based on that indicated above, these signatures show that cancer cells utilize massive fatty acids to meet the demand of cell membrane synthesis, mainly for lipid raft and lipid-modified signaling molecules (79); and a large fraction of their membrane lipids are biosynthesized de novo rather than scavenging from extracellular sources. In de novo lipogenesis, fatty acid synthase (FAS) catalyzes the synthesis of palmitate from acetyl-CoA or malonyl-CoA in the presence of NADPH as a redox equivalent. FAS expression is commonly low in non-proliferating cells that typically import lipids from the extracellular milieu. In contrast, actively proliferating cells, especially tumor cells, have increased demands for lipids, which is highly dependent on de novo synthesis. So FAS is frequently upregulated in many types of tumors (80-82) including gastric cancer $(83,84)$; and increased FAS expression is linked to tumor proliferation, chemoresistance and poorer prognosis in cancers (85-88). Thus, this key enzyme implicated in lipogenesis has been studied as potential target in anti-neoplastic therapy (84). On the other hand, enhancement of fatty acid- $\beta$ oxidation is also considered to be an important metabolic reprogramming in the early stage of some cancer types (89), as it produces more ATP and acetyl coenzyme A which in turn can accelerate the rate of citric acid oxidation and serve as the energy source (90). Furthermore, production of polyunsaturated fatty acids, to some extent, is also associated with tumor cell proliferation, apoptosis and angiogenesis $(91,92)$.

Nucleotide metabolism. Tumor cells are in a state of such rapid proliferation and differentiation that frequent nucleotide synthesis and metabolism are upregulated significantly. Accumulation of the end products of nucleotide catabolism is characterized by the higher levels of uric acid or urate $(25,30)$ in gastric cancer patients or animal models. Other purines compounds like hypoxanthine and guanosine were also increased $(35,37)$, but Aa et al showed decreases in uridine (an RNA building block) (28). Nucleotides are also associated with energy metabolism, mainly in the form of ATP and GTP. Of tumor cells, adequate energy should be supplied to meet their proliferation. In this way, it is assumed that nucleotide phosphates should increase in cancer tissues compared with normal tissues. However, Hirayama and colleagues (35), identified that there was no noticeable difference between gastric cancer tissues and adjacent normal tissues with regard to most nucleotide phosphates (ATP, ADP, GTP, and GDP), total adenylate and energy charge. Accordingly, it infers that cancer cells gain growth superiority over their normal counterparts by switching metabolic patterns of energy to anaerobic glycolysis and possibly fumarate respiration that we have discussed above, instead of securing more ATP.

Other altered metabolisms. Except for the changed metabolisms mentioned above, other metabolite concentrations also show increased or decreased trend in the development of gastric cancer. Increased level of creatinine, a waste product of muscle metabolism, was detected in urine samples of tumor groups (33), which might be induced by lower total body skeletal mass among cachectic patients $(93,94)$. Changes in inositol level of gastric malignancy patients are investigated in either tumor tissues $(28,36,37)$ or urine samples $(30)$, but its mechanism and significance are poorly understood.

\section{Metabolomics in diagnosis, treatment and prognostic prediction of gastric cancer}

Diagnosis. Early diagnosis is the key element determining the outcome of treatment in cancer research, but current application of cancer biomarkers, endoscopy and imaging is still not satisfactory. Serum biomarkers, like CEA and CA19-9, are not effective given their poor sensitivity or specificity. Inconsistent diagnostic efficacy at endoscopy that results from the variations in skill and experience of endoscopists and pathologist might lead to missed diagnosis at early phase, while positive results displayed on imaging examination (such as barium meal and computer tomography) are prone to advanced stage. Interestingly, utility of various -omics technologies open a new field to discover potential biomarkers for gastric cancer diagnosis, especially based on metabolomics. 
Exploration of gastric cancer biomarkers in blood or urine is more appreciated because of its non-invasive priority. Yu et al demonstrated that metabolic profiles were quite different in gastric cancer patients with different pathological types in the Correa model, but intestinal metaplasia shared similar metabolic phenotype (threonate, glutamate and azelaic acid) in plasma with neoplastic groups $(25,95)$. Ikeda et al also identified that there were obvious variations in serum metabolic profiles of gastrointestinal cancers (including esophageal, gastric and colorectal) in contrast to healthy volunteers (27). In particular, changes in the levels of 3-hydroxypropionic acid and pyruvic acid were sufficient to differentiate gastric cancer from esophageal and colorectal cancer, and showed high values for both sensitivity (84.6 and 70.0\%) and specificity (71.4 and $90.0 \%$ ) compared with conventional biomarkers (CA19-9 and CEA) (27). The diagnostic potential of serum metabolic profiles between gastric cancer and non-cancer groups was also confirmed by Song et al, and these alterations occurred at early stage of gastric carcinogenesis (26).

Recently, one urine metabolomics in gastric cancer found that 14 out of 17 metabolites detected from training set (94 urine samples) via GC-MS showed diagnostic value better than classic blood biomarkers on validation set (199 urine samples) (34). Six of them (L-alanine, L-isoleucine, L-serine, L-threonine, L-proline and L-methionine) revealed satisfactory diagnostic values with the area under the ROC of $>0.75$. Chan et al also revealed that gastric cancer has a unique urine metabolic profiles in contrast to benign gastric diseases and healthy patients, especially 2 -hydroxyisobutyrate, 3 -indoxylsulfate and alanine, producing a discriminatory model with the area under the curve (AUC) of 0.95 (33). Another study reported that metabolites altered in urinary data of gastric cancer patients was predicted with higher sensitivity than CA19-9 and CEA (31).

In tissue testing, $\mathrm{Wu}$ and colleagues indicated that 18 metabolites were detected differently between the malignant tissues and the adjacent non-malignant tissues of gastric mucosa with AUC value of 0.9629 (37), but tissue testing was not a non-invasive approach in contrast to blood or urine testing. Our data, on the other hand, showed that higher levels of tyrosine, phenylalanine and tryptophan in the gastric juice were detected in the early phase of gastric carcinogenesis (40), and the sensitivity and specificity for gastric cancer detection with phenylalanine was 87.9 and $79.4 \%$ respectively (41).

Metastasis and prognosis. Most gastric cancer-related deaths occur as a result of metastasis, even among patients undergoing gastrectomy. Unfortunately, no molecular markers for predicting metastasis and prognosis are accessible.

Based on metabolomics, $\mathrm{Wu}$ and colleagues showed that five metabolites (increased L-cysteine, hypoxanthine and L-tyrosine; decreased phenanthrenol and butanoic acid) were detected differently between non-invasive (T1 and T2) and invasive (T3 and T4) groups, furthermore, 4-hydroxyphenylacetate, alanine, phenylacetylglycine, mannitol, glycolate and arginine levels were significantly correlated with cancer $\mathrm{T}$ stage (37). By establishing animal models with gastric cancer cell line SGC-7901, Chen et al confirmed that metabolites correlated to proline and serine metabolism could distinguish metastatic from non-metastatic specimens with an AUC value of 1.0 (36). Study conducted by $\mathrm{Hu}$ et al suggested that decreased levels of alanine, glycerol, L-proline, butanoic acid and L-threonic acid as well as increased levels of butanediotic acid and myo-inositol could detect non-metastatic and metastatic groups (AUC=1.00) (30).

Significantly, Chen and coworkers recently evaluated the prognostic value of 17 urinary metabolites, which have been identified differently between gastric cancer group and normal group, by following up 82 out of 112 gastric cancer cases for 3-5 years after surgery (34). They discovered that patients with higher levels of proline, p-cresol and 4-hydroxybenzoic acid display poor prognosis with median survival time 16, 15 and 15 months, respectively. Furthermore, the concentration of p-cresol closely correlated with gastric cancer stage, which was gradually increased with the stage of the patients.

It is possible that changes in proline might be essential in tumor metastasis. As we have mentioned above, proline in tumor tissues might result from the degradation of collagen (73). This process mainly begins with the activation of MMPs and degradation of microenvironmental ECM, which partially accounts for the tumor invasion and metastasis (96). In this respective, elevated proline serving as metastatic biomarker for gastric cancer is possible, but further research is necessary.

Treatment. Chemosensitivity prediction that aims to maximize the therapeutic response and minimize adverse effects is a difficult task in the treatment of advanced tumors. One of classical approaches for predicting the activity of anticancer agents is cell culture testing, which is mainly based on clone formation, cell metabolic activity assays, proliferation and tumor growth in vitro experiments. However, it must be noted that these methods still fail to fully reproduce the tumor microenvironment, although current patient-derived primary cell culture or patient-derived tumor xenograft models are able to retain cellular heterogeneity of original tumors (97).

Lu et al, in particular, suggested that some conventional cytotoxic anticancer agents (vincristine, taxol, 5-fluorouracil, doxorubicin, cisplatin, camptothecin) lost their efficacy apparently when cultured PNAC-1 cells (pancreatic cancer) in vitro were deprived of glucose (98). Similarly, a recent study also identified that high glucose conditions promoted SGC-7901 proliferation in vitro and reduced chemosensitivity in vivo or in vitro (99). We could speculate that responses of gastric cancer against anticancer drugs in actual microenvironment in vivo might be considerably different from what we expect in culture condition. Therefore, utilizing metabolomics is considered to be a promising tool to assess the sensitivity of chemotherapy in virtual conditions and discovering therapeutic targets regarding specific tumor metabolism $(20,21)$.

Wang et al applied high performance liquid chromatography coupled with a quadrupole time-of-flight mass spectrometer to predict chemotherapy response in a human xenograft model of gastric cancer administered with cisplatin plus 5-fluorouracil (5-FU) (100). Consequently, 1-acyl-lysophosphatidylcholine and polyunsaturated fatty acid were proposed to surveil gastric cancer chemosensitivity, since 1-acyl-lysophosphatidylcholine can regulate the activity of enzymes like phospholipase A2 (PLA2) and lysophosphatidylcholine acetyltransferases. PLA2 catalyzes the production 
of arachidonic acid that is likely to promote cell cycle arrest and apoptosis dependent on ceramide pathway $(101,102)$, while lysophosphatidylcholine acetyltransferases catalyzes phospholipid synthesis linked to tumor cell proliferation. Another study suggested that proline was reduced while glutamate increased dramatically, and PRODH (catalyzes the metabolic production of glutamate from proline proceeds) mRNA expression was upregulated 2-fold after 5-FU administration; but they were less affected in 5-FU-resistant cells (103). Thus PRODH might make it possible to be a marker for assessing intracellular dynamic responses to 5-FU. Additionally, Kim and colleagues utilized ${ }^{1} \mathrm{H}-\mathrm{NMR}$ to investigate the metabolic changes in urine sample following Adriamycin (ADR) treatment for gastric adenocarcinoma in an animal model (104). This study revealed that levels of trimethylamine oxide, hippurate and taurine, which all decreased in tumor group without treatment, were increased dramatically after ADR disposal; while 2-oxoglutarate, 3-indoxylsulfate, trigonelline, trimethylamine and citrate recovered to those of normal group (104). Alterations in these metabolites might be ascribed to the pharmacological activity of ADR that activates apoptotic process of gastric cancer cells via ADR-induced genotoxic stress.

In another study, dysregulation of pyruvic acid efflux in gastric cardia cancer was observed with the combination of proteomics and metabolomics (38). Furthermore, Cai et al also found that downregulation of lactate dehydrogenase $\mathrm{A}$ (LDH-A) and overexpression of pyruvate dehydrogenase B (PDH-B) could force pyruvic acid into the Krebs cycle rather than the glycolysis process in gastric cancer cell line AGS, consequently inhibiting cell growth and migration (38). In view of the above, LDH or PDH might serve as a therapeutic target in gastric cancer treatment.

\section{Current perspectives and future directions}

As we indicated above, cumulative studies employing metabolomics have yielded initial and promising results in gastric cancer research. However, inconsistent results across studies can be observed, probably because of the different sensitivity of metabolomics methods (105), variety of experimental subjects (patients, animal models or in vitro cell culture), and the number of samples. Additionally, values of those biomarkers should be further validated with larger cohorts and normalized metabolomics analysis. Furthermore, it should be noted that investigations targeted at the mechanism of the altered metabolism and specific metabolic pathways in gastric cancer are relatively deficient at present, so it is difficult to draw a clear dividing line on metabolism for common cancers and this disease based on a handful of studies that looked also at the role of metabolomics. Overall, exploring the metabolic disorders and gastric carcinogenesis still has far to go.

On the other hand, metabolomics locate at the downstream of genomics, transcriptomics and proteomics, mapping the complete metabolic changes under specific conditions associated with pathogenic factors, host or environmental co-effectors. However, it is essential to combine metabolomics with other -omics methods to get a more integrated understanding of gastric carcinogenesis (Fig. 1). For instance, metabolomic genome-wide association studies (mGWAS) have their priority in quantifying metabolic data and uncovering genetic variants affecting metabolite levels (106). Impacts of the microbiome on the metabolome are also an area of increasing interest, because perturbation of gastrointestinal microbiota composition or function including Helicobacter pylori has been proved to play a role in gastric carcinogenesis $(107,108)$. Furthermore, microbederived metabolites also produce effects on cancer cells, such as butanoic acid. Some research revealed that it can modulate immune response via the differentiation of colonic regulatory T cells (109) and inhibit colonic tumor cells $(110,111)$, although the signaling mechanism was not clearly understood. Thus, it can explain the fact that some changed metabolites in gastric cancer such as butanoic acid (37), mannitol (37) and p-cresol (34) that are commonly thought of artificial substances, can originate from fermentation by microorganism in gastric flora. Given this, it is reasonable to presume that gastric flora might be incorporated into an in-depth study of the prominent disorders of metabolism in gastric cancer, but there is still a gap in further research.

\section{Conclusions}

Gastric cancer is one of the most malignant tumors worldwide, and remains a major global health threat. Though its pathogenesis is unknown, promising discoveries have been made with the emergence of -omics studies. Most strikingly, metabolomics provides us in-depth information on metabolic perturbation between healthy and neoplastic states in the stomach, and further help us discovery disease-specific biomarkers. As technology advances and our understanding of metabolic perturbation in gastric cancer grows, new diagnostic and therapeutic targets will undoubtedly emerge. Ultimately, these advances can be translated into clinical practice to realize the goal of truly personalized cancer treatment.

\section{Acknowledgements}

This study was supported by National Natural Science Foundation of China (no. 81672410).

\section{References}

1. Siegel RL, Miller KD and Jemal A: Cancer statistics, 2016. CA Cancer J Clin 66: 7-30, 2016.

2. Conteduca V, Sansonno D, Lauletta G, Russi S, Ingravallo G and Dammacco F: H. pylori infection and gastric cancer: State of the art (Review). Int J Oncol 42: 5-18, 2013.

3. Amieva M and Peek RM Jr: Pathobiology of Helicobacter pyloriinduced gastric cancer. Gastroenterology 150: 64-78, 2016.

4. Kim J, Yum S, Kang C and Kang SJ: Gene-gene interactions in gastrointestinal cancer susceptibility. Oncotarget 7: 67612-67625, 2016.

5. Raei N,Behrouz B,Zahri S and Latifi-Navid S: Helicobacter pylori infection and dietary factors act synergistically to promote gastric cancer. Asian Pac J Cancer Prev 17: 917-921, 2016.

6. Warburg O: On respiratory impairment in cancer cells. Science 124: 269-270, 1956.

7. Hanahan D and Weinberg RA: Hallmarks of cancer: The next generation. Cell 144: 646-674, 2011.

8. Yun J, Rago C, Cheong I, Pagliarini R, Angenendt P, Rajagopalan H, Schmidt K, Willson JK, Markowitz S, Zhou S, et al: Glucose deprivation contributes to the development of KRAS pathway mutations in tumor cells. Science 325: 1555-1559, 2009.

9. Xu W, Yang H, Liu Y, Yang Y, Wang P, Kim SH, Ito S, Yang C, Wang P, Xiao MT, et al: Oncometabolite 2-hydroxyglutarate is a competitive inhibitor of $\alpha$-ketoglutarate-dependent dioxygenases. Cancer Cell 19: 17-30, 2011. 
10. Dang L, White DW, Gross S, Bennett BD, Bittinger MA, Driggers EM, Fantin VR, Jang HG, Jin S, Keenan MC, et al: Cancer-associated IDH1 mutations produce 2-hydroxyglutarate. Nature 465: 966, 2010.

11. Fischer K, Hoffmann P, Voelkl S, Meidenbauer N, Ammer J, Edinger M, Gottfried E, Schwarz S, Rothe G, Hoves S, et al: Inhibitory effect of tumor cell-derived lactic acid on human T cells. Blood 109: 3812-3819, 2007.

12. Dietl K, Renner K, Dettmer K, Timischl B, Eberhart K, Dorn C, Hellerbrand C, Kastenberger M, Kunz-Schughart LA, Oefner PJ et al: Lactic acid and acidification inhibit TNF secretion and glycolysis of human monocytes. J Immunol 184: 1200-1209, 2010.

13. Herber DL, Cao W, Nefedova Y, Novitskiy SV, Nagaraj S, Tyurin VA, Corzo A, Cho HI, Celis E, Lennox B, et al: Lipid accumulation and dendritic cell dysfunction in cancer. Nat Med 16: $880-886,2010$.

14. Zhang J, Ahn WS, Gameiro PA, Keibler MA, Zhang Z and Stephanopoulos G: 13C isotope-assisted methods for quantifying glutamine metabolism in cancer cells. Methods Enzymol 542: 369-389, 2014.

15. Beger RD: A review of applications of metabolomics in cancer Metabolites 3: 552-574, 2013

16. Putri SP, Yamamoto S, Tsugawa $\mathrm{H}$ and Fukusaki E: Current metabolomics: Technological advances. J Biosci Bioeng 116 9-16, 2013.

17. Duarte IF, Diaz SO and Gil AM: NMR metabolomics of human blood and urine in disease research. J Pharm Biomed Anal 93 17-26, 2014.

18. Ussher JR, Elmariah S, Gerszten RE and Dyck JR: The emerging role of metabolomics in the diagnosis and prognosis of cardiovascular disease. J Am Coll Cardiol 68: 2850-2870, 2016.

19. Guma M, Tiziani S and Firestein GS: Metabolomics in rheumatic diseases: Desperately seeking biomarkers. Nat Rev Rheumatol 12: 269-281, 2016

20. Herrmann K, Walch A, Balluff B, Tänzer M, Höfler H, Krause BJ, Schwaiger M, Friess H, Schmid RM and Ebert MP: Proteomic and metabolic prediction of response to therapy in gastrointestinal cancers. Nat Clin Pract Gastroenterol Hepatol 6: 170-183, 2009.

21. Armitage EG and Southam AD: Monitoring cancer prognosis, diagnosis and treatment efficacy using metabolomics and lipidomics. Metabolomics 12: 146, 2016.

22. Jayavelu ND and Bar NS: Metabolomic studies of human gastric cancer (Review). World J Gastroenterol 20: 8092-8101, 2014.

23. Chan AW, Gill RS, Schiller D and Sawyer MB: Potential role of metabolomics in diagnosis and surveillance of gastric cancer. World J Gastroenterol 20: 12874-12882, 2014.

24. Miyagi Y, Higashiyama M, Gochi A, Akaike M, Ishikawa T, Miura T, Saruki N, Bando E, Kimura H, Imamura F, et al: Plasma free amino acid profiling of five types of cancer patients and its application for early detection. PLoS One 6: e24143, 2011.

25. Yu L, Aa J, Xu J, Sun M, Qian S, Cheng L, Yang S and Shi R: Metabolomic phenotype of gastric cancer and precancerous stages based on gas chromatography time-of-flight mass spectrometry. J Gastroenterol Hepatol 26: 1290-1297, 2011.

26. Song H, Peng JS, Dong-Sheng Y, Yang ZL, Liu HL, Zeng YK, Shi XP and Lu BY: Serum metabolic profiling of human gastric cancer based on gas chromatography/mass spectrometry. Braz J Med Biol Res 45: 78-85, 2012.

27. Ikeda A, Nishiumi S, Shinohara M, Yoshie T, Hatano N, Okuno T, Bamba T, Fukusaki E, Takenawa T, Azuma T, et al: Serum metabolomics as a novel diagnostic approach for gastrointestinal cancer. Biomed Chromatogr 26: 548-558, 2012.

28. Aa J, Yu L, Sun M, Liu L, Li M, Cao B, Shi J, Xu J, Cheng L, Zhou J, et al: Metabolic features of the tumor microenvironmen of gastric cancer and the link to the systemic macroenvironment. Metabolomics 8: 164-173, 2012.

29. Choi JM, Park WS, Song KY, Lee HJ and Jung BH: Development of simultaneous analysis of tryptophan metabolites in serum and gastric juice - an investigation towards establishing a biomarker test for gastric cancer diagnosis. Biomed Chromatogr 30: 1963-1974, 2016

30. Hu JD, Tang HQ, Zhang Q, Fan J, Hong J, Gu JZ and Chen JL: Prediction of gastric cancer metastasis through urinary metabolomic investigation using GC/MS. World J Gastroenterol 17: 727-734, 2011.

31. Jung J, Jung Y, Bang EJ, Cho SI, Jang YJ, Kwak JM, Ryu DH, Park S and Hwang GS: Noninvasive diagnosis and evaluation of curative surgery for gastric cancer by using NMR-based metabolomic profiling. Ann Surg Oncol 21 (Suppl 4): S736-S742, 2014.
32. Liang Q, Wang C and Li B: Metabolomic analysis using liquid chromatography/mass spectrometry for gastric cancer. Appl Biochem Biotechnol 176: 2170-2184, 2015.

33. Chan AW, Mercier P, Schiller D, Bailey R, Robbins S, Eurich DT, Sawyer MB and Broadhurst D: (1)H-NMR urinary metabolomic profiling for diagnosis of gastric cancer. Br J Cancer 114: 59-62, 2016.

34. Chen Y, Zhang J, Guo L, Liu L, Wen J, Xu L, Yan M, Li Z, Zhang X, Nan P, et al: A characteristic biosignature for discrimination of gastric cancer from healthy population by high throughput GC-MS analysis. Oncotarget 7: 87496-87510, 2016.

35. Hirayama A, Kami K, Sugimoto M, Sugawara M, Toki N, Onozuka H, Kinoshita T, Saito N, Ochiai A, Tomita M, et al: Quantitative metabolome profiling of colon and stomach cancer microenvironment by capillary electrophoresis time-of-flight mass spectrometry. Cancer Res 69: 4918-4925, 2009.

36. Chen JL, Tang HQ, Hu JD, Fan J, Hong J and Gu JZ: Metabolomics of gastric cancer metastasis detected by gas chromatography and mass spectrometry. World J Gastroenterol 16: 5874-5880, 2010.

37. Wu H, Xue R, Tang Z, Deng C, Liu T, Zeng H, Sun Y and Shen X: Metabolomic investigation of gastric cancer tissue using gas chromatography/mass spectrometry. Anal Bioanal Chem 396: 1385-1395, 2010

38. Cai Z, Zhao JS, Li JJ, Peng DN, Wang XY, Chen TL, Qiu YP, Chen PP, Li WJ, Xu LY, et al: A combined proteomics and metabolomics profiling of gastric cardia cancer reveals characteristic dysregulations in glucose metabolism. Mol Cell Proteomics 9: 2617-2628, 2010

39. Song H, Wang L, Liu HL, Wu XB, Wang HS, Liu ZH, Li Y, Diao DC, Chen HL and Peng JS: Tissue metabolomic fingerprinting reveals metabolic disorders associated with human gastric cancer morbidity. Oncol Rep 26: 431-438, 2011.

40. Deng K, Lin S, Zhou L, Geng Q, Li Y, Xu M and Na R: Three aromatic amino acids in gastric juice as potential biomarkers for gastric malignancies. Anal Chim Acta 694: 100-107, 2011

41. Deng K, Lin S, Zhou L, Li Y, Chen M, Wang Y and Li Y: High levels of aromatic amino acids in gastric juice during the early stages of gastric cancer progression. PLoS One 7: e49434, 2012.

42. Koukourakis MI, Pitiakoudis M, Giatromanolaki A, Tsarouha A Polychronidis A, Sivridis E and Simopoulos C: Oxygen and glucose consumption in gastrointestinal adenocarcinomas: Correlation with markers of hypoxia, acidity and anaerobic glycolysis. Cancer Sci 97: 1056-1060, 2006.

43. Pedersen PL, Mathupala S, Rempel A, Geschwind JF and Ko YH: Mitochondrial bound type II hexokinase: A key player in the growth and survival of many cancers and an ideal prospect for therapeutic intervention. Biochim Biophys Acta 1555: 14-20, 2002.

44. Gatenby RA and Gillies RJ: Why do cancers have high aerobic glycolysis? Nat Rev Cancer 4: 891-899, 2004.

45. Yuan LW, Yamashita H and Seto Y: Glucose metabolism in gastric cancer: The cutting-edge. World J Gastroenterol 22: 2046-2059, 2016.

46. Israelsen WJ and Vander Heiden MG: Pyruvate kinase: Function, regulation and role in cancer. Semin Cell Dev Biol 43: 43-51, 2015.

47. $\mathrm{Wu} \mathrm{J}, \mathrm{Hu} \mathrm{L}$, Chen M, Cao W, Chen $\mathrm{H}$ and He T: Pyruvate kinase M2 overexpression and poor prognosis in solid tumors of digestive system: Evidence from 16 cohort studies. Onco Targets Ther 9: 4277-4288, 2016.

48. Augoff K, Hryniewicz-Jankowska A and Tabola R: Lactate dehydrogenase 5: An old friend and a new hope in the war on cancer. Cancer Lett 358: 1-7, 2015.

49. Le A, Cooper CR, Gouw AM, Dinavahi R, Maitra A, Deck LM, Royer RE, Vander Jagt DL, Semenza GL and Dang CV: Inhibition of lactate dehydrogenase A induces oxidative stress and inhibits tumor progression. Proc Natl Acad Sci USA 107: 2037-2042, 2010.

50. Dhup S, Dadhich RK, Porporato PE and Sonveaux P: Multiple biological activities of lactic acid in cancer: Influences on tumor growth, angiogenesis and metastasis. Curr Pharm Des 18: 1319-1330, 2012

51. Lardner A: The effects of extracellular $\mathrm{pH}$ on immune function. J Leukoc Biol 69: 522-530, 2001.

52. Gottfried E, Kunz-Schughart LA, Ebner S, Mueller-Klieser W, Hoves S, Andreesen R, Mackensen A and Kreutz M: Tumorderived lactic acid modulates dendritic cell activation and antigen expression. Blood 107: 2013-2021, 2006.

53. Chen Z, Lu W, Garcia-Prieto $C$ and Huang P: The Warburg effect and its cancer therapeutic implications. J Bioenerg Biomembr 39: 267-274, 2007. 
54. Costello LC and Franklin RB: 'Why do tumour cells glycolyse?' From glycolysis through citrate to lipogenesis. Mol Cell Biochem 280: 1-8, 2005

55. Lu Y, Zhang X, Zhang H, Lan J, Huang G, Varin E, Lincet H, Poulain L and Icard P: Citrate induces apoptotic cell death: A promising way to treat gastric carcinoma? Anticancer Res 31: 797-805, 2011

56. Weljie AM and Jirik FR: Hypoxia-induced metabolic shifts in cancer cells: Moving beyond the Warburg effect. Int J Biochem Cell Biol 43: 981-989, 2011.

57. Ichinoe M, Yanagisawa N, Mikami T, Hana K, Nakada N, Endou H, Okayasu I and Murakumo Y: L-Type amino acid transporter 1 (LAT1) expression in lymph node metastasis of gastric carcinoma: Its correlation with size of metastatic lesion and Ki-67 labeling. Pathol Res Pract 211: 533-538, 2015.

58. Zhang M, Zhu GY, Gao HY, Zhao SP and Xue Y: Expression of tissue levels of matrix metalloproteinases and tissue inhibitors of metalloproteinases in gastric adenocarcinoma. J Surg Oncol 103: 243-247, 2011

59. Sampieri CL, León-Córdoba K and Remes-Troche JM: Matrix metalloproteinases and their tissue inhibitors in gastric cancer as molecular markers. J Cancer Res Ther 9: 356-363, 2013.

60. Qian HR and Yang Y: Functional role of autophagy in gastric cancer. Oncotarget 7: 17641-17651, 2016.

61. Hensley CT, Wasti AT and DeBerardinis RJ: Glutamine and cancer: Cell biology, physiology, and clinical opportunities. $\mathrm{J}$ Clin Invest 123: 3678-3684, 2013.

62. Nicklin P, Bergman P, Zhang B, Triantafellow E, Wang H, Nyfeler B, Yang H, Hild M, Kung C, Wilson C, et al: Bidirectiona transport of amino acids regulates mTOR and autophagy. Cell 136: 521-534, 2009.

63. Liu W, Le A, Hancock C, Lane AN, Dang CV, Fan TW and Phang JM: Reprogramming of proline and glutamine metabolism contributes to the proliferative and metabolic responses regulated by oncogenic transcription factor c-MYC. Proc Nat Acad Sci USA 109: 8983-8988, 2012.

64. Possemato R, Marks KM, Shaul YD, Pacold ME, Kim D Birsoy K, Sethumadhavan S, Woo HK, Jang HG, Jha AK, et al: Functional genomics reveal that the serine synthesis pathway is essential in breast cancer. Nature 476: 346-350, 2011.

65. Godin-Ethier J, Hanafi LA, Piccirillo CA and Lapointe R Indoleamine 2,3-dioxygenase expression in human cancers: Clinical and immunologic perspectives. Clin Cancer Res 17 6985-6991, 2011

66. Wiggins T, Kumar S, Markar SR, Antonowicz S and Hanna GB Tyrosine, phenylalanine, and tryptophan in gastroesophageal malignancy: A systematic review. Cancer Epidemiol Biomarkers Prev 24: 32-38, 2015.

67. Bauer TM, Jiga LP, Chuang JJ, Randazzo M, Opelz G and Terness P: Studying the immunosuppressive role of indoleamine 2,3-dioxygenase: Tryptophan metabolites suppress rat allogeneic T-cell responses in vitro and in vivo. Transpl Int 18: 95-100, 2005

68. Zhang R, Li H, Yu J, Zhao J, Wang X, Wang G, Yao Z, Wei F, Xue Q and Ren X: Immunoactivative role of indoleamine 2,3-dioxygenase in gastric cancer cells in vitro. Mol Med Rep 4: 169-173, 2011.

69. McGaha TL, Huang L, Lemos H, Metz R, Mautino M, Prendergast GC and Mellor AL: Amino acid catabolism: A pivotal regulator of innate and adaptive immunity. Immunol Rev 249: 135-157, 2012.

70. Jain M, Nilsson R, Sharma S, Madhusudhan N, Kitami T, Souza AL, Kafri R, Kirschner MW, Clish CB and Mootha VK Metabolite profiling identifies a key role for glycine in rapid cancer cell proliferation. Science 336: 1040-1044, 2012

71. Rose ML, Madren J, Bunzendahl H and Thurman RG: Dietary glycine inhibits the growth of B16 melanoma tumors in mice. Carcinogenesis 20: 793-798, 1999.

72. Amin K, Li J, Chao WR, Dewhirst MW and Haroon ZA: Dietary glycine inhibits angiogenesis during wound healing and tumor growth. Cancer Biol Ther 2: 173-178, 2003.

73. Bruns H, Petrulionis M, Schultze D, Al Saeedi M, Lin S, Yamanaka K, Ambrazevičius M, Strupas K and Schemmer P. Glycine inhibits angiogenic signaling in human hepatocellular carcinoma cells. Amino Acids 46: 969-976, 2014.

74. Bruns H, Kazanavicius D, Schultze D, Saeedi MA, Yamanaka K, Strupas K and Schemmer P: Glycine inhibits angiogenesis in colorectal cancer: Role of endothelial cells. Amino Acids 48 : 2549-2558, 2016

75. Phang JM, Donald SP, Pandhare J and Liu Y: The metabolism of proline, a stress substrate, modulates carcinogenic pathways. Amino Acids 35: 681-690, 2008
76. Agustsson T, Rydén M, Hoffstedt J, van Harmelen V, Dicker A, Laurencikiene J, Isaksson B, Permert J and Arner P: Mechanism of increased lipolysis in cancer cachexia. Cancer Res 67: 5531-5537, 2007

77. Raghavamenon A, Garelnabi M, Babu S, Aldrich A, Litvinov D and Parthasarathy S: Alpha-tocopherol is ineffective in preventing the decomposition of preformed lipid peroxides and may promote the accumulation of toxic aldehydes: A potential explanation for the failure of antioxidants to affect human atherosclerosis. Antioxid Redox Signal 11: 1237-1248, 2009.

78. Schallreuter KU and Wood JM: Azelaic acid as a competitive inhibitor of thioredoxin reductase in human melanoma cells. Cancer Lett 36: 297-305, 1987.

79. Muñoz-Pinedo C,El Mjiyad N and Ricci JE: Cancer metabolism: Current perspectives and future directions. Cell Death Dis 3: e248, 2012.

80. Swinnen JV, Roskams T, Joniau S, Van Poppel H, Oyen R, Baert L, Heyns W and Verhoeven G: Overexpression of fatty acid synthase is an early and common event in the development of prostate cancer. Int J Cancer 98: 19-22, 2002.

81. Flavin R, Peluso S, Nguyen PL and Loda M: Fatty acid synthase as a potential therapeutic target in cancer. Future Oncol 6: $551-562,2010$

82. Hao Q, Li T, Zhang X, Gao P, Qiao P, Li S and Geng Z: Expression and roles of fatty acid synthase in hepatocellular carcinoma. Oncol Rep 32: 2471-2476, 2014.

83. Kusakabe T, Nashimoto A, Honma K and Suzuki T: Fatty acid synthase is highly expressed in carcinoma, adenoma and in regenerative epithelium and intestinal metaplasia of the stomach. Histopathology 40: 71-79, 2002.

84. Ito T, Sato K, Maekawa H, Sakurada M, Orita H, Shimada K, Daida H, Wada R, Abe M, Hino O, et al: Elevated levels of serum fatty acid synthase in patients with gastric carcinoma. Oncol Lett 7: 616-620, 2014

85. Lin HP, Cheng ZL, He RY, Song L, Tian MX, Zhou LS, Groh BS, Liu WR, Ji MB, Ding C, et al: Destabilization of fatty acid synthase by acetylation inhibits de novo lipogenesis and tumor cell growth. Cancer Res 76: 6924-6936, 2016.

86. Takahiro T, Shinichi K and Toshimitsu S: Expression of fatty acid synthase as a prognostic indicator in soft tissue sarcomas. Clin Cancer Res 9: 2204-2212, 2003.

87. Menendez JA, Lupu R and Colomer R: Inhibition of tumorassociated fatty acid synthase hyperactivity induces synergistic chemosensitization of HER-2/ neu-overexpressing human breast cancer cells to docetaxel (taxotere). Breast Cancer Res Treat 84 183-195, 2004

88. Duan J, Sun L, Huang H, Wu Z, Wang L and Liao W: Overexpression of fatty acid synthase predicts a poor prognosis for human gastric cancer. Mol Med Rep 13: 3027-3035, 2016.

89. Khasawneh J, Schulz MD, Walch A, Rozman J, Hrabe de Angelis M, Klingenspor M, Buck A, Schwaiger M, Saur D, Schmid RM, et al: Inflammation and mitochondrial fatty acid beta-oxidation link obesity to early tumor promotion. Proc Natl Acad Sci USA 106: 3354-3359, 2009.

90. Liu Y: Fatty acid oxidation is a dominant bioenergetic pathway in prostate cancer. Prostate Cancer Prostatic Dis 9: 230-234, 2006.

91. Hyde CA and Missailidis S: Inhibition of arachidonic acid metabolism and its implication on cell proliferation and tumourangiogenesis. Int Immunopharmacol 9: 701-715, 2009.

92. Lu X, Yu H, Ma Q, Shen S and Das UN: Linoleic acid suppresses colorectal cancer cell growth by inducing oxidant stress and mitochondrial dysfunction. Lipids Health Dis 9: 106, 2010.

93. Swaminathan R, Major P, Snieder H and Spector T: Serum creatinine and fat-free mass (lean body mass). Clin Chem 46 1695-1696, 2000.

94. Eisner R, Stretch C, Eastman T, Xia J, Hau D, Damaraju S, Greiner R, Wishart D and Baracos V: Learning to predict cancerassociated skeletal muscle wasting from ${ }^{1} \mathrm{H}-\mathrm{NMR}$ profiles of urinary metabolites. Metabolomics 7: 25-34, 2011

95. Correa P: Human gastric carcinogenesis: A multistep and multifactorial process - First American Cancer Society Award Lecture on Cancer Epidemiology and Prevention. Cancer Res 52: 6735-6740, 1992

96. Brown GT and Murray GI: Current mechanistic insights into the roles of matrix metalloproteinases in tumour invasion and metastasis. J Pathol 237: 273-281, 2015.

97. Lau V, Wong AL, Ng C, Mok Y, Lakshmanan M and Yan B: Drug sensitivity testing platforms for gastric cancer diagnostics. J Clin Pathol 69: 93-96, 2016. 
98. Lu J, Kunimoto S, Yamazaki Y, Kaminishi M and Esumi $\mathrm{H}$ Kigamicin D, a novel anticancer agent based on a new antiausterity strategy targeting cancer cells' tolerance to nutrient starvation. Cancer Sci 95: 547-552, 2004.

99. Zhao W, Chen R, Zhao M, Li L, Fan L and Che XM: High glucose promotes gastric cancer chemoresistance in vivo and in vitro. Mol Med Rep 12: 843-850, 2015.

100. Wang X, Yan SK, Dai WX, Liu XR, Zhang WD and Wang JJ: A metabonomic approach to chemosensitivity prediction of cisplatin plus 5-fluorouracil in a human xenograft model of gastric cancer. Int J Cancer 127: 2841-2850, 2010.

101. Ilsley JN, Nakanishi M, Flynn C, Belinsky GS, De Guise S, Adib JN, Dobrowsky RT, Bonventre JV and Rosenberg DW: Cytoplasmic phospholipase A2 deletion enhances colon tumorigenesis. Cancer Res 65: 2636-2643, 2005.

102. Ganesan K, Ivanova T, Wu Y, Rajasegaran V, Wu J, Lee MH, Yu K, Rha SY, Chung HC, Ylstra B, et al: Inhibition of gastric cancer invasion and metastasis by PLA2G2A, a novel betacatenin/TCF target gene. Cancer Res 68: 4277-4286, 2008.

103. Sasada S, Miyata Y, Tsutani Y, Tsuyama N, Masujima T, Hihara J and Okada M: Metabolomic analysis of dynamic response and drug resistance of gastric cancer cells to 5-fluorouracil. Oncol Rep 29: 925-931, 2013.

104. Kim KB, Yang JY, Kwack SJ, Kim HS, Ryu DH, Kim YJ, Bae JY, Lim DS, Choi SM, Kwon MJ, et al: Potential metabolomic biomarkers for evaluation of adriamycin efficacy using a urinary ${ }^{1} \mathrm{H}-\mathrm{NMR}$ spectroscopy. J Appl Toxicol 33: 1251-1259, 2013.

105. Büscher JM, Czernik D, Ewald JC, Sauer U and Zamboni N: Cross-platform comparison of methods for quantitative metabolomics of primary metabolism. Anal Chem 81: 2135-2143, 2009.
106. Adamski J and Suhre K: Metabolomics platforms for genome wide association studies - linking the genome to the metabolome. Curr Opin Biotechnol 24: 39-47, 2013.

107. Lofgren JL, Whary MT, Ge Z, Muthupalani S, Taylor NS, Mobley M, Potter A, Varro A, Eibach D, Suerbaum S, et al: Lack of commensal flora in Helicobacter pylori-infected INS-GAS mice reduces gastritis and delays intraepithelial neoplasia. Gastroenterology 140: 210-220, 2011.

108. Lertpiriyapong K, Whary MT, Muthupalani S, Lofgren JL, Gamazon ER, Feng Y, Ge Z, Wang TC and Fox JG: Gastric colonisation with a restricted commensal microbiota replicates the promotion of neoplastic lesions by diverse intestinal microbiota in the Helicobacter pylori INS-GAS mouse model of gastric carcinogenesis. Gut 63: 54-63, 2014.

109. Furusawa Y, Obata Y, Fukuda S, Endo TA, Nakato G, Takahashi D, Nakanishi Y, Uetake C, Kato K, Kato T, et al: Commensal microbe-derived butyrate induces the differentiation of colonic regulatory T cells. Nature 504: 446-450, 2013.

110. Vanhoutvin SA, Troost FJ, Hamer HM, Lindsey PJ, Koek GH, Jonkers DM, Kodde A, Venema K and Brummer RJ: Butyrateinduced transcriptional changes in human colonic mucosa. PLoS One 4: e6759, 2009.

111. Singh N, Gurav A, Sivaprakasam S, Brady E, Padia R, Shi H, Thangaraju M, Prasad PD, Manicassamy S, Munn DH, et al: Activation of Gpr109a, receptor for niacin and the commensal metabolite butyrate, suppresses colonic inflammation and carcinogenesis. Immunity 40: 128-139, 2014. 